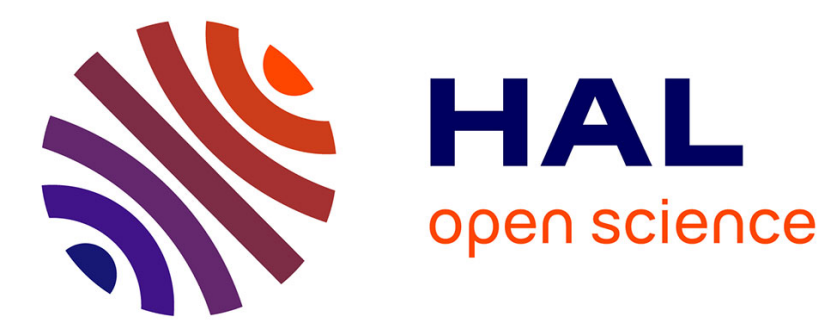

\title{
Plasma potential and opacity calculations
}

\author{
W. Jarrah, J.-C. Pain, D. Benredjem
}

\section{To cite this version:}

W. Jarrah, J.-C. Pain, D. Benredjem. Plasma potential and opacity calculations. High Energy Density Physics, 2019, 32, pp.8 - 13. 10.1016/j.hedp.2019.04.006 . hal-03484524

\section{HAL Id: hal-03484524 https://hal.science/hal-03484524}

Submitted on 20 Dec 2021

HAL is a multi-disciplinary open access archive for the deposit and dissemination of scientific research documents, whether they are published or not. The documents may come from teaching and research institutions in France or abroad, or from public or private research centers.
L'archive ouverte pluridisciplinaire HAL, est destinée au dépôt et à la diffusion de documents scientifiques de niveau recherche, publiés ou non, émanant des établissements d'enseignement et de recherche français ou étrangers, des laboratoires publics ou privés.

\section{다)(1) $(5$}

Distributed under a Creative Commons Attribution - NonCommercial| 4.0 International 


\title{
Plasma potential and opacity calculations
}

\author{
W. Jarrah ${ }^{a}$, J.-C. Pain ${ }^{b}$, D. Benredjem ${ }^{a}$ \\ ${ }^{a}$ Laboratoire Aimé Cotton (CNRS, Université Paris-Sud, ENS Paris-Saclay), \\ Orsay, France \\ ${ }^{b} C E A, D A M$, DIF, F-91297 Arpajon, France
}

\begin{abstract}
The knowledge of the radiative properties of hot plasmas is crucial when we study the absorption in the ablator of ICF capsules. For this purpose, we developed a new code that includes a collisional-radiative module. Atomic data, calculated by either Cowan or FAC code, is the input.

In a previous work we investigated the absorption of $\mathrm{C}-\mathrm{Si}$ mixtures, where the silicon is a dopant. Atomic data was obtained by Cowan's code, and the ionization potential depression was given by the Stewart and Pyatt formula. Classical semiempirical formulas for the cross-sections of the various processes occurring in a hot and dense plasma were modified with the help of correction factors in order to match the FAC results. In this work we present the ion charge distribution and compare the calculated transmission of a pure silicon plasma to a recent experiment. We also investigate the effect of the radiation field on the opacity.
\end{abstract}

\section{INTRODUCTION}

The knowledge of the opacity and emissivity is very important when one deals with the radiative properties of ICF and astrophysical plasmas. The aim of this work is the calculation of the opacity and the comparison with experimental results. In a first calculation we compare the opacity of a pure silicon plasma to a recent experiment. In a second calculation, we study a mixture of carbon and silicon and investigate the role of the radiation field of the Hohhlram on the population kinetics. This means that processes like photoexcitation and photoionization are taken into account.

We recently developed a Non Local Thermodynamic Equilibrium (NLTE) code which accounts for most collisional and radiative processes. The atomic database is obtained by the FAC code [1]. It consists in a large set of level energies and spontaneous emission rates. The cross-sections of the collisional and radiative processes are treated by modified semi-empirical formulas. For 
example, for the collisional excitation cross-section we started from the formula of Sampson and Zhang [2,3]. The Sampson and Zhang cross-section is multiplied by a function in order to retrieve accurate cross-sections given by the FAC code. The effect of the radiation field is modeled with a Planckian distribution, yielding rates whose effect on the population kinetics is investigated. Line broadening, line shift due to screening effects and Ionization Potential Depression (IPD) are also taken into account.

In Sec. 2, we describe a collisional-radiative model. The NLTE code is used to calculate the opacity. The atomic database of carbon and silicon involving various ionic stages is provided by the FAC code. The collisional excitation cross-sections are obtained by fitting the values given by the FAC code with a Van Regemorter-Sampson-Zhang-like analytical formula [2, 3], yielding a new Gaunt factor. Specific Gaunt factors are also derived for photoionization and collisional ionization cross-sections. The effect of the radiation field on the population kinetics is also examined.

In Sec. 3 we compare our calculations for a pure silicon plasma to experimental results [4] and to calculations using a different approach. In fact, for low- or mid- $Z$ dopants the number of spectral lines is usually tractable. In such cases, we are able to perform a detailed-line-accounting (DLA) calculation. Unfortunately, when the number of transitions is large this approach becomes prohibitive. An alternative hybrid approach, based on a detailed description whenever it is required and a statistical one when a large number of lines overlap, is proposed by a CEA group [5]. Such a model enables one to obtain precise opacities that are useful in the context of astrophysics and ICF plasmas. The detailed (fine-structure) opacity code SCO-RCG $[5,6,7]$ enables one to compute precise opacities for the calculation of accurate Rosseland means. It is a reliable tool for the diagnostics and interpretation of spectroscopy experiments in Local Thermodynamic Equilibrium (LTE) plasmas as well as for the study of the intrinsic properties of complex atomic spectra. It uses limited computational resources and is setup for automatic execution, making the generation of opacity tables easier. The configurations are generated by an adapted version of the SCO code [8] on the basis of a statistical fluctuation theory and a self-consistent computation of atomic structure is performed for all the configurations. In such a way, each configuration has its own set of wavefunctions. The latter are determined in a single-configuration approximation. One peculiarity of the code is that it does not rely on the "isolated atom" picture, but on a realistic atom-in-plasma modelling (equation of state). Relativistic effects are taken into account in the Pauli approximation. The DLA part of the spectrum is obtained using an adapted version of the RCG routine from Cowan's suite of atomic structure and spectra codes [9]. The RCG source code was used for decades by spectroscopists, it has many available options and is well documented. The data required for the calculation of the detailed transition arrays (Slater, spin-orbit and dipolar integrals) are obtained from SCO, providing in this way a consistent description of the 
plasma screening effects on the wavefunctions. Then, the level energies and the lines are calculated by RCG.

The computation starts with an average-atom calculation in LTE, which provides the average populations of the subshells. The LTE fluctuation theory [10] around the average-atom non-integer populations is used in order to determine the range of variation of the populations. The configurations are then sorted according to their Boltzmann weights, estimated using the averageatom wavefunctions. A DLA calculation is performed if possible and necessary for all the transition arrays; DLA computations are carried out only for pairs of configurations giving rise to less than 800,000 lines. In other cases, transition arrays are represented by Gaussian profiles in the UTA (Unresolved Transition Array [11, 12]) or SOSA (Spin-Orbit Split Array [13]) formalisms. The code involves coalescence criteria in order to decide whether a detailed treatment of the lines is necessary and to determine the validity of the statistical methods. The loosely-bound subshells are gathered in a so-called "Rydberg" supershell. The populations of those states may be small, but their number is so huge that they can play a significant role in the opacity. The orbitals in the Rydberg supershell are chosen in a way so that they weakly interact with inner orbitals. In the present case, the amount of detailed calculations performed in SCO-RCG is largely dominant. The PRTA (Partially Resolved Transition Array) model [14] was recently implemented. It enables one to replace many statistical transition arrays by small-scale DLA calculations. The model was extended to the STA formalism [15], consisting in omitting the Rydberg supershell in the computation, and adding its contribution to the widths of all lines. The contribution of the Rydberg supershell is included as a Gaussian "dressing function" [6]. The strength of this approach is that it enables one to take into account many highly excited states and satellite lines.

\section{MODEL}

\section{Atomic structure}

We have calculated the level energies and the radiative spontaneous emission rates with the FAC code. This code generates a set of atomic levels, as well as the cross-sections for collisional excitation, collisional ionization, photoionization and auto-ionization. FAC uses the Dirac-Fock method in $j j$-coupling in which the relativistic effects are taken into account. The atomic code requires a relevant set of electronic configurations. The knowledge of these configurations relies on the Quantum Average Atom model, where the bound electrons are treated in the quantum theory frame. The free electrons are treated within the semi-classical Thomas-Fermi model.

The perturbers are responsible for a plasma potential that affects the 
energy level values of the active ions. In fact, the plasma potential yields a reduction of the binding energy. This is the IPD effect. In a previous work [16], we have taken into account the IPD effect by using the well-known formula of Stewart and Pyatt [17]. However, as shown by Hoarty et al [18], this formula as well as the alternative formula of Ecker and Kröll [19] can under or overestimate the IPD. In this work, the plasma potential is calculated within the Thomas-Fermi model, inside the Wigner sphere surrounding the considered ion. The charge neutrality is assumed inside the sphere [20]. Also, free electrons as well as ion perturbers are responsible for line shifts in the emission and absorption spectra. We have used the formula of Massacrier and Dubau for line shift [21]:

$$
\Delta E\left(n l-n^{\prime} l^{\prime}\right)=-\frac{2}{3} \pi N_{e}\left(<r^{2}>_{n^{\prime} l^{\prime}}-<r^{2}>_{n l}\right)
$$

and

$$
<r^{2}>_{n l}=\frac{n^{2}}{2 z^{2}}\left[5 n^{2}+1-3 l(l+1)\right] a_{0}^{2},
$$

where $N_{e}$ is the free-electron density and $\left\langle r^{2}\right\rangle$ the average square radius at zero free electron density. $a_{0}$ is the Bohr radius and $z$ the ion charge. A recent work on line shift [22] gives a similar expression (Eqs 6, 7) for H-like ions. However the reliability of such formulas is still an open question and subject of debate due to the lack of experimental data. The Stark effect associated with the electric field generated by the neighboring ions may also induce a shift which is certainly smaller that the shift due to free electrons [23].

\section{Atomic processes}

In this work, we account for spontaneous emission, induced emission, collisional excitation, collisional ionization, autoionization, photoionization and the inverse processes: photoexcitation, collisional deexcitation, 3-body recombination, dielectronic recombination and radiative recombination. We use empirical formulas for some processes. In fact, we modified existing empirical formula in order to retrieve the accurate cross-sections obtained by the FAC code. For example, if we focus on collisional excitation, we start from the formula of Van Regemorter giving the cross-section for excitation from atomic level $i$ to atomic level $j[24]$ :

$$
\sigma_{i j}=\frac{8 \pi}{\sqrt{3}}\left(\frac{1}{E}\right)^{2} \frac{f_{i j}}{\Delta E_{i j}} \pi a_{0}^{2} \bar{g},
$$

where $E$ and $\Delta E_{i j}$ are respectively the incident electron and the transition energies in units of Rydbergs. $f_{i j}$ is the oscillator strength and $\bar{g}$ is the Gaunt factor proposed by Van Regemorter. This formula presents two causes of error. It does not account for the optically forbidden transitions, and may under or overestimate the optically allowed ones. Sampson and Zhang [2, 3] proposed 
the following Gaunt factor for $\Delta n \geq 1$ transitions in H-like ions:

$$
\begin{aligned}
\bar{g}_{\mathrm{SZ}}(x) & =\frac{\sqrt{3}}{2 \pi}\left\{\ln x+H_{n}\left(1-\frac{1}{x}\right)\left[\left(\frac{\Delta E_{i j}}{\chi_{i}}\right)^{r_{n}^{\prime}}+A_{n}\left(\frac{\Delta E_{i j}}{\chi_{i}}-1\right)\right]\right\} \\
& +0,31 n\left(\frac{\Delta E_{i j}}{\chi_{i}}\right) \frac{1}{x}
\end{aligned}
$$

where $x$ is the incident free-electron energy in units of the transition energy, i.e. $x=E / \Delta E_{i j}$, and $\chi_{i}$ the ionization potential of the electron that is being collisionally excited. $H_{n}, A_{n}$ and $r_{n}^{\prime}$ are parameters given in Table 1of Ref. [3].

Table 1: Sampson and Zhang parameters

\begin{tabular}{ccccccccccc}
\hline & $n=1$ & $n=2$ & $n=3$ & $n=4$ & $n=5$ & $n=6$ & $n=7$ & $n=8$ & $n=9$ & $n=10$ \\
\hline$H_{n}$ & 1.48 & 3.64 & 5.93 & 8.32 & $2.15 \mathrm{n}$ & $2.15 \mathrm{n}$ & $2.15 \mathrm{n}$ & $2.15 \mathrm{n}$ & $2.15 \mathrm{n}$ & $2.15 \mathrm{n}$ \\
\hline$A_{n}$ & 1.3 & 0.59 & 0.38 & 0.286 & 0.229 & 0.192 & 0.164 & 0.141 & 0.121 & 0.105 \\
\hline$r_{n}^{\prime}$ & 2.2 & 1.9 & 1.73 & 1.65 & 1.6 & 1.56 & 1.54 & 1.52 & 1.52 & 1.52 \\
\hline
\end{tabular}

Unfortunately, the Gaunt factor $\bar{g}_{\mathrm{SZ}}$ yields large discrepancies with the accurate cross-sections obtained by the FAC code. To show the discrepancies let us concentrate on collisional excitation. In Fig. 1, we compare the crosssection given by Eqs. 3-4 to the cross-section obtained by the FAC code. We can see that the discrepancy is large. In order to agree with the accurate cross-section given by FAC, we define a new Gaunt factor:

$$
\bar{g}_{\mathrm{DW}}=\bar{g}_{\mathrm{SZ}} \times g_{\mathrm{CE}}(x),
$$

where $g_{\mathrm{CE}}(x)=A \ln x+B . A$ and $B$ are parameters depending on the chemical element, the initial subshell of the excited electron and on whether the ion is hydrogenic or not. The parameters are adapted so that the new cross-section fits FAC cross-sections. The same procedure is applied to the other processes listed above.

The effect of the radiation field on the opacity is also investigated, so that photoionization, photoexcitation and their inverse processes are taken into account. The photoionization cross-sections $\sigma_{\mathrm{PI}}$ obtained by the FAC code are fitted by simple formulas. For example, the $1 s$ photoionization in $\mathrm{H}$-like $\mathrm{C}$ is well represented by the following formula, as shown in Fig. 2:

$$
\sigma_{\mathrm{PI}}(x) \simeq a_{3} x^{3}+a_{2} x^{2}+a_{1} x+a_{0}+a_{-1} x^{-1}+a_{-2} x^{-2}+a_{-3} x^{-3},
$$

where $x$ is the energy of the incident photon in ionization potential (IP) units, and $a_{i}$ parameters that are calculated for each photoionization transition. 


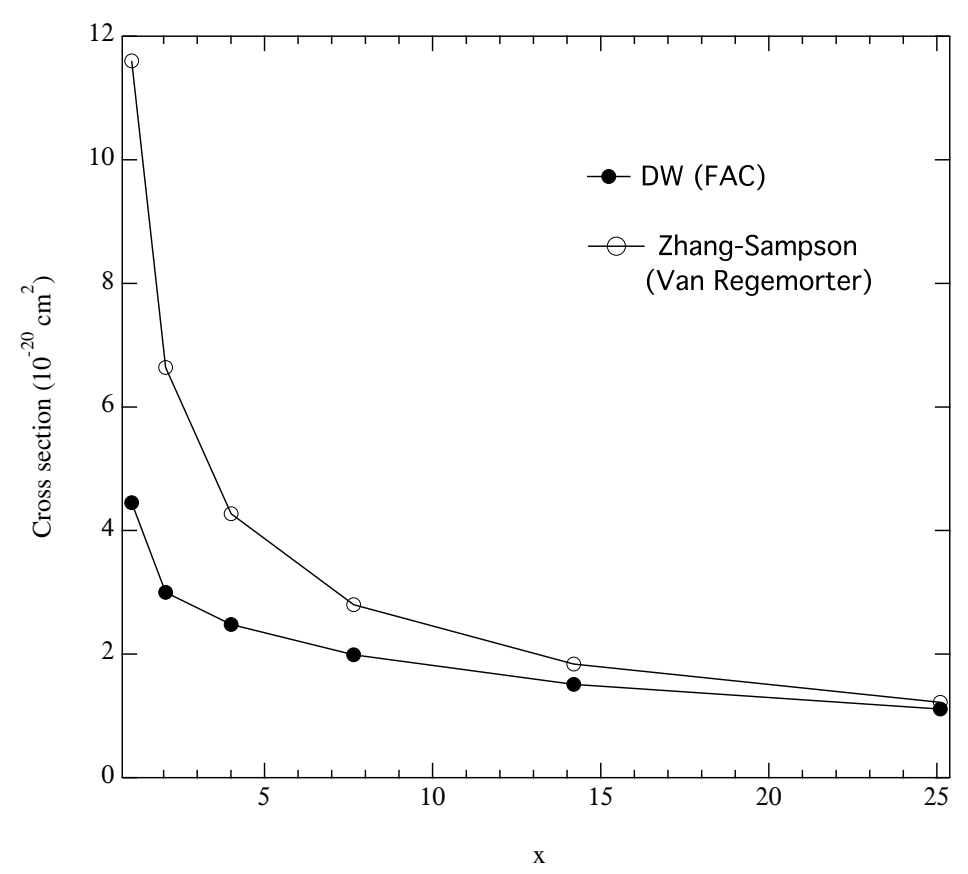

Fig. 1. Collisional excitation cross-section of the transition $3 p-4 s$ in $\mathrm{Si}^{13+}$ as a function of the incident electron energy in units of the transition energy.

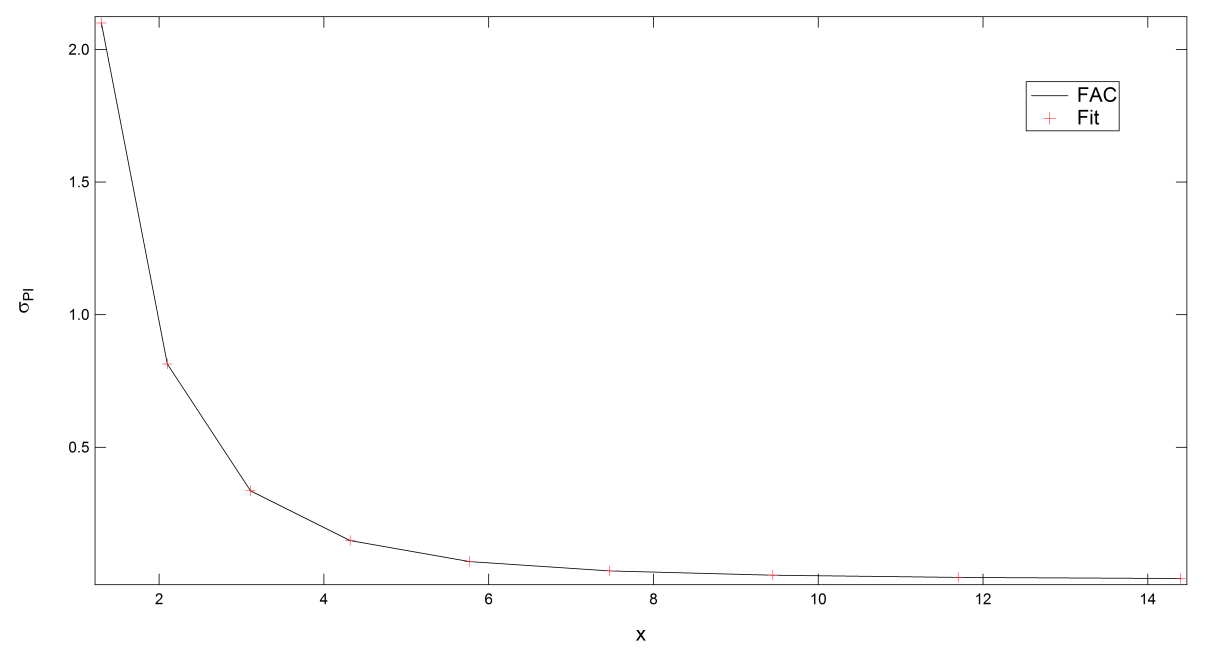

Fig. 2. Photoionization cross-section of the 1 s electron in $\mathrm{C}^{5+}$ as a function of the photon energy in units of the transition energy.

All our fitting formulas provide cross-sections that agree very well with FAC results. Because collisional-radiative equations (Sec. 3) are time consuming, it is much easier to use empirical formulas than running the FAC code. These are then more appropriate for extensive calculations. 


\section{Populations}

Level populations and ionic fractions depend strongly on the electronic temperature and density. When collisional deexcitation overcomes spontaneous emission the plasma is in LTE. The populations are then given by the Boltzmann equation and the Saha-Boltzmann equation. In NLTE it is necessary to develop a collisional-radiative model (CR). To be efficient the CR calculations necessitates reliable rates for the numerous atomic processes that occur between levels. This approach generally requires a large amount of calculation. The steady state approximation is valid when the plasma conditions vary slowly with respect to the time of interest.

If the LTE approximation is not fulfilled, the populations of the various levels $i$ of ions of charge $z$, named $n_{z, i}$, with $0 \leq z \leq Z$, where $Z$ is the atomic number, are governed by the following rate equations:

$$
\frac{d n_{z, i}}{d t}=\sum_{i^{\prime}, z^{\prime}}\left[n_{z^{\prime}, i^{\prime}} W_{\left(z^{\prime}, i^{\prime}\right) \rightarrow(z, i)}-n_{z, i} W_{(z, i) \rightarrow\left(z^{\prime}, i^{\prime}\right)}\right]
$$

where the $W^{\prime}$ 's designate rates of atomic processes: $W_{\left(z^{\prime}, i^{\prime}\right) \rightarrow(z, i)}$ is responsible for an increase of the level $i$ population while $W_{(z, i) \rightarrow\left(z^{\prime}, i^{\prime}\right)}$ is responsible for a population decrease. We assume steady-state plasmas, i.e.,

$$
\frac{d n_{z, i}}{d t}=0
$$

In the present work we are mainly interested in the interpretation of spectroscopy experiments involving ns-pulse laser plasmas. Since the characteristic times of atomic processes are much smaller than the hydrodynamic time scales, the stationarity assumption is justified.

We have taken into account spontaneous emission, collisional excitation, collisional ionization, photoionization, autoionization and the corresponding inverse processes according to the detailed balance.

\section{Opacity}

We account for bound-bound $(b b)$, bound-free $(b f)$ and free-free $(f f)$ transitions. The bound-bound opacity is calculated within DLA, according to which the absorption coefficient $\left(\right.$ in $\mathrm{cm}^{-1}$ ) reads:

$$
k_{b b}(E)=\frac{h^{3} c^{2}}{8 \pi} \sum_{z} \sum_{j \in z, i \in z(j<i)} n_{z, j} \frac{g_{i}}{g_{j}} \frac{A_{i j}}{\left(E_{i}-E_{j}\right)^{2}} \phi_{i j}(E),
$$

where $g$ designates the statistical weight, $E$ the energy level, $A$ the spontaneous emission rate of the transition $i \rightarrow j$ and $\phi$ the absorption line profile. The 
double sum involves all the ion stages and all allowed transitions.

Concerning the $b f$ contribution to the opacity, we used the online version of GIPPER (General Ionization Processes in the Presence of Electrons and Radiation) [25] to generate ionization potential tables. We then used the Kramers' approximation [26] in the Detailed-Configuration-Accounting (DCA) frame to calculate the bound-free absorption :

$$
k_{b f}(E)=\sum_{z} \sum_{C \in z} n_{C}^{z} \sum_{n l \in C} \frac{64}{3 \sqrt{3}} \pi \alpha a_{0}^{2} \frac{P_{n l} g_{b f}}{Z_{\mathrm{eff}}(n l)} \frac{\sqrt{\mathrm{Ry}} \chi_{n l}^{2.5}}{E^{3}},
$$

where $\chi_{n l}$ is the ionization potential of the $n l$ subshell in the configuration $C$ which belongs to the ion stage $z$ and $g_{b f}$ a new Gaunt factor associated with the photoionization cross-section. Equation 10 is valid only if the photon energy is larger than $\chi_{n l}$. Otherwise, $k_{b f}=0 . P_{n l}$ is the number of electrons occupying the subshell $n l, n_{z, C}=\sum_{i \in C} n_{z, i}$ the population of the electronic configuration $C$ and $Z_{\text {eff }}(n l)$ the screened nuclear charge seen by an electron on subshell $n l$ [27]. On the other hand, FAC is able to calculate many ion stages at a time. The ionization energy in this case is simply the energy difference between the initial and final states of the ionization. We thus used the DLA to calculate the bound-free absorption when using FAC photoionization cross-sections.

Finally, the $f f$ absorption coefficient is given by:

$$
k_{f f}(E)=\frac{16}{3} \sqrt{\frac{2}{3}} \pi^{5 / 2} \frac{e^{6} \hbar^{2}}{c m_{e}^{3 / 2}} \frac{N_{e}}{\left(k_{B} T_{e}\right)^{1 / 2}} \frac{1}{E^{3}}\left(1-\mathrm{e}^{-\frac{E}{k_{B} T_{e}}}\right) \sum_{z} z^{2} N_{z} .
$$

The free-free Gaunt factor $g_{f f}$ is taken equal to 1 .

\section{RESULTS}

\section{Comparison to experiment}

In this section, we compare our calculations to the experiment of Xiong et al. [4], in which the transmission of a silicon plasma was measured at an electron temperature $T_{\mathrm{e}}=72 \pm 5 \mathrm{eV}$ and a density $\rho=(6.0 \pm 1.2) \mathrm{mg} / \mathrm{cm}^{3}$, corresponding to $N_{\mathrm{e}} \simeq 1.3 \times 10^{21} \mathrm{~cm}^{-3}$.

Figure 3 represents the ionic fractions of silicon. We compare our calculations to the experimental results and calculations with the SCO-RCG code. Our results are in good agreement with those of Xiong et al. However, we can point out some discrepancies: Xiong et al. obtained $46.85 \%$ for the fraction of Be-like Si while our calculation gives $42 \%$. The mean ion charge presents a good agreement: we obtained $\bar{Z}=9.88$ while Xiong et al. found $\bar{Z}=10.07$. The hybrid approach shows discrepancies with our calculations and the results of 
Xiong et al. as well.

In Fig. 4, we show the calculated and the measured transmission. Our NLTE approach shows a better agreement with experiment than SCO-RCG. However, some discrepancies in line positions occur for some lines in $\mathrm{Si}^{8+}$ and $\mathrm{Si}^{11+}$ ions. This could be overcome by a better treatment of the line shift. Also, for both calculations there is an underestimation of the absorption between 1800 and $1810 \mathrm{eV}$. This can be explained by the absence of a few configurations in our calculations. Figure 10 of Ref. [4] suggest that the authors were faced with the same problem, when using the ATOMIC code as well as their own DTA (Detail Term Accounting) code.

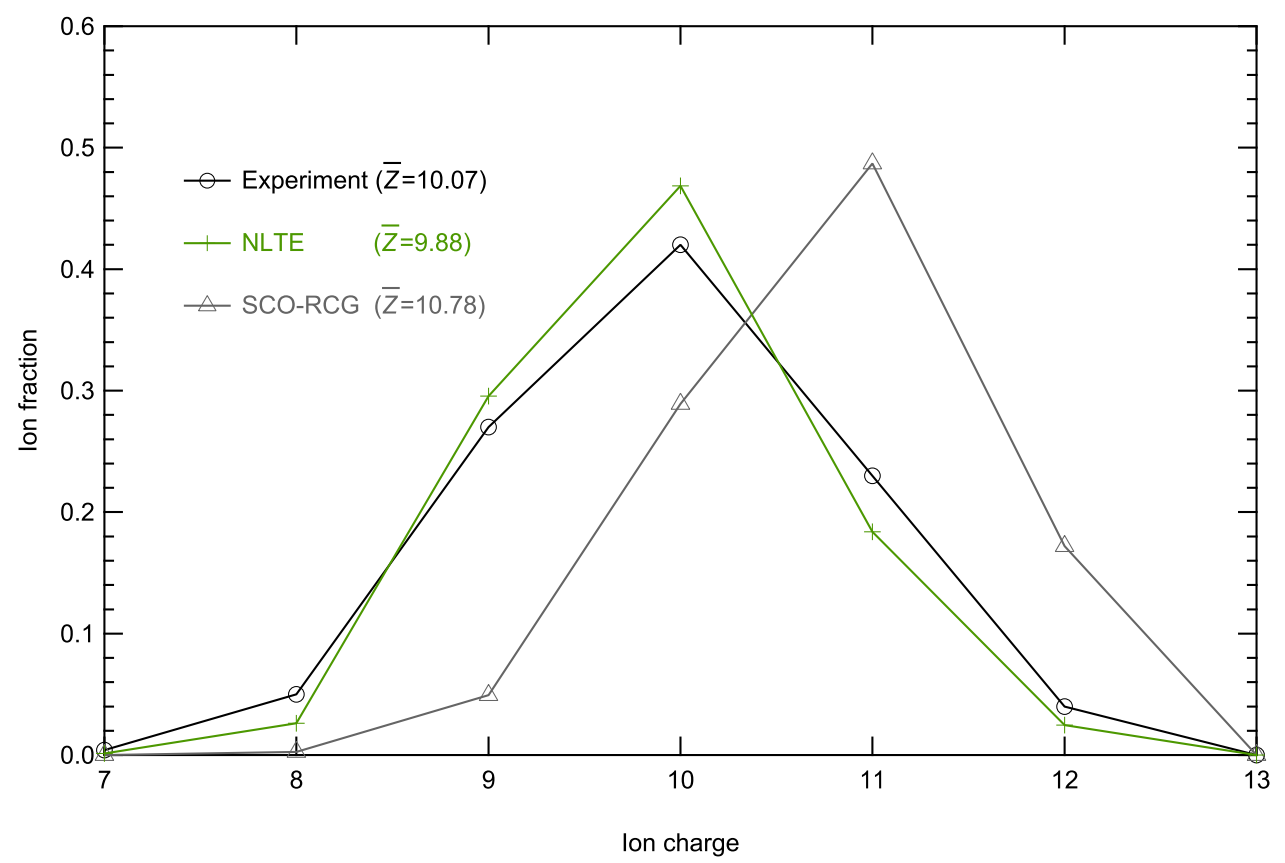

Fig. 3. Ionic fractions of a silicon plasma at $T_{\mathrm{e}}=72 \mathrm{eV}$ and $N_{\mathrm{e}}=1.3 \times 10^{21} \mathrm{~cm}^{-3}$.

We notice that our calculated spectrum contains the main structures with a good agreement as concerns transmission levels and line energies. However, some differences are still present, in particular around 1829 and $1853 \mathrm{eV}$, where we overestimate the amplitude of absorption lines, and around $1819 \mathrm{eV}$ where our calculated peak is shifted to the high-energy side.

Since, in SCO-RCG, each configuration has its own set of wavefunctions, determined within a self-consistent potential in the ion-cell model, the line energies should be rather well calculated. However, SCO-RCG fails to reproduce the intensities of several structures (for instance around $1850 \mathrm{eV}$ ). This can not be explained by the use of statistical methods UTA or SOSA, since in the present calculation all the transition arrays are treated in a detailed way. The fact that the code described in Ref. [16] provides a better description of those structures may come from NLTE effects or from configuration interaction, which is treated only to a limited extent in SCO-RCG. Indeed, in the 


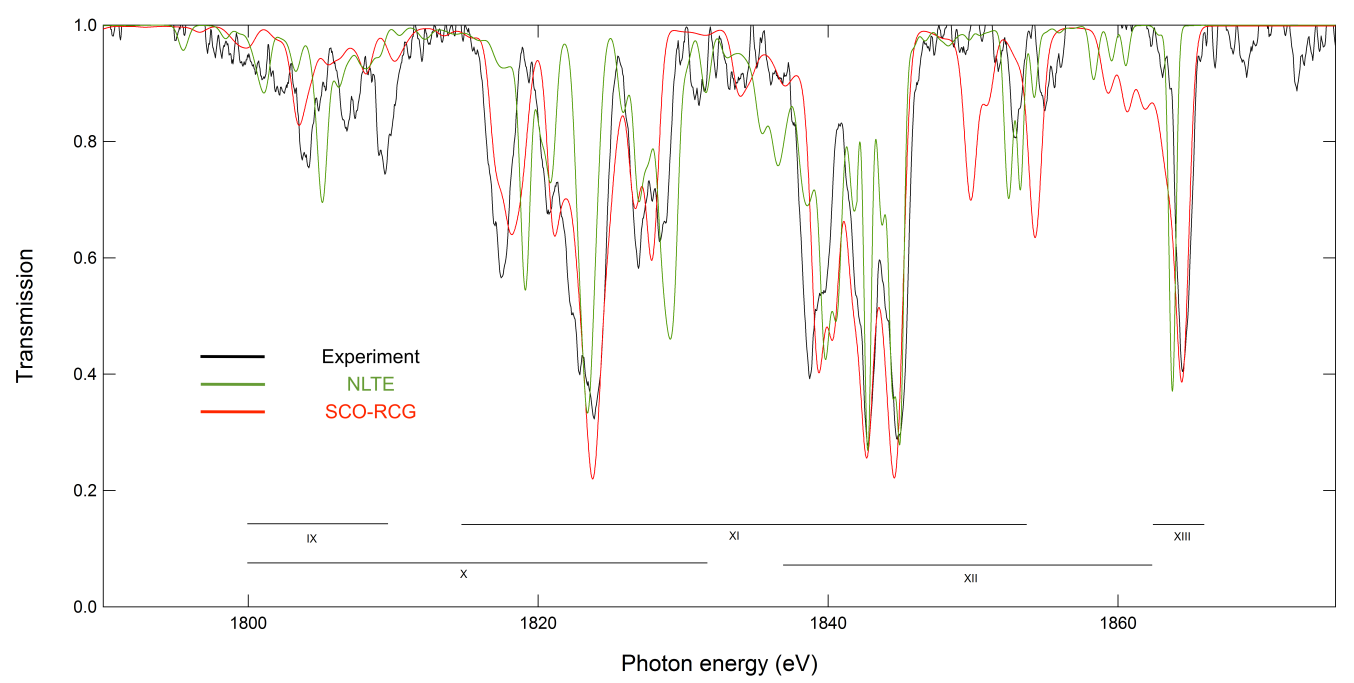

Fig. 4. Transmission of a silicon plasma. Plasma parameters, as in Fig. 3.

latter code, configuration interaction is restricted to relativistic configurations belonging to the same non-relativistic parent configuration, which is not the case of our code described in Ref. [16]. In particular, it was recently pointed out by Li et al. that configuration interaction between $1 s^{1} 2 s^{2} n \ell$ and $1 s 2 p^{2} n \ell$ in Be-like ions was particularly strong; this may have a significant impact in the spectral range of the experiment [28]. We plan to investigate that possibility in the future.

\section{Role of the radiation field}

We now focus on a mixture of carbon and silicon. We have used our NLTE code to calculate the opacity and to investigate the importance of the radiation field on the opacity spectrum. The opacity of a mixture is defined by :

$$
k(\nu)=p_{\mathrm{C}} k_{\mathrm{C}}+p_{\mathrm{Si}} k_{\mathrm{Si}},
$$

where $p_{\mathrm{Si}}$ is the mass fraction of the dopant. In our study, $p_{\mathrm{Si}}=0.05$ and then $p_{\mathrm{C}}=0.95$. The radiation field emitted by the Hohlraum can affect the ionic populations as well as the level populations, especially when the collision processes become less important, e.g. at low density and high temperature. The emission spectra of the gold plasma evolves with time as the ablation occurs and the properties of the plasma change. However, it is often approached by a Planck's distribution with a radiation temperature $T_{r}=300 \mathrm{eV}[29,30]$. We calculated the rates of the photoexcitation and photoionization by using a Planck distribution. The rates are then used in Eq. 7. A more accurate emission spectra can be obtained by using our collisional-radiative code. This alternative dramatically increases the amount of calculations required for opacity. This should be necessary at lower densities. Indeed in that case the radiative processes compete with collisional processes. 
In Fig. 5 we show the absorption coefficient of the $\mathrm{C}-\mathrm{Si}$ mixture. The electron temperature and density are fixed to $300 \mathrm{eV}$ and $6 \times 10^{23} \mathrm{~cm}^{-3}$, respectively. The radiation temperature is assumed identical to the electron temperature. The case where the radiation field is omitted is represented by the legend $T_{r}=0$. We can see that the radiation field has a negligible effect. This is not surprising since the plasma conditions are close to LTE and, as expected, the effect of the radiation field on the atomic populations is negligible.

At the lower density $N_{e}=2 \times 10^{23} \mathrm{~cm}^{-3}$, the plasma is no longer in LTE. The radiative processes may affect the populations and thus the opacity. In Fig. 6 we show the absorption coefficient of the mixture. We can see that the radiation field has a small effect on the $b b$ contribution of $\mathrm{Si}$, in the range [1950-1980] eV.

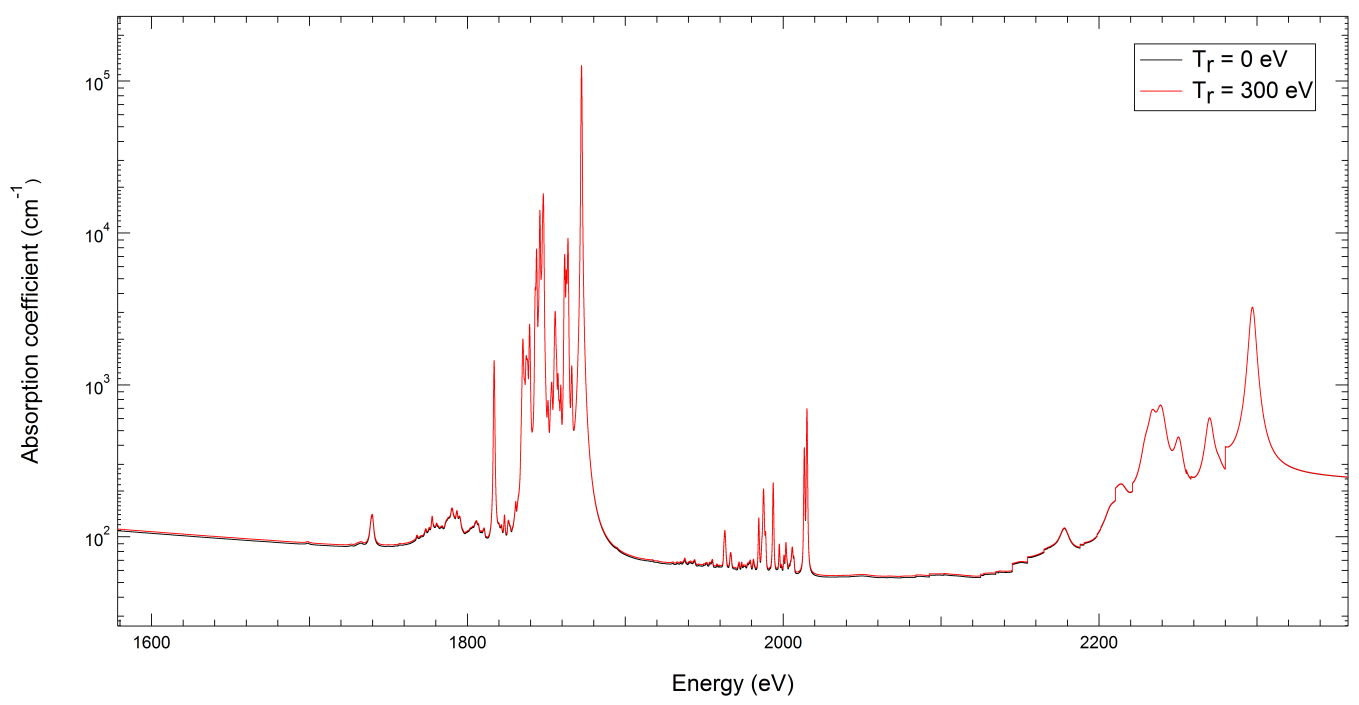

Fig. 5. Effect of the radiation field on the absorption of the $\mathrm{C}-\mathrm{Si}$ mixture. $T_{e}=300$ $\mathrm{eV}$ and $N_{e}=6 \times 10^{23} \mathrm{~cm}^{-3}$.

\section{CONCLUDING REMARKS}

We have calculated the opacity of a pure silicon plasma and compared our results to a recent experiment and to other calculations. We have also calculated the opacity of a mixture where the dopant is silicon. In the second study we have shown that the radiation field has a very small effect on the population kinetics. The calculations are performed by using a NLTE code developed at Laboratoire Aimé Cotton. The cross-sections of the various processes are fitted with empirical formula that involve new Gaunt factors. In a future work we will extend the atomic database of the dopant. To maintain the amount of calculation at a reasonable level, it would be necessary to treat statistically some intervals in the $b b$ regions of the dopant. 


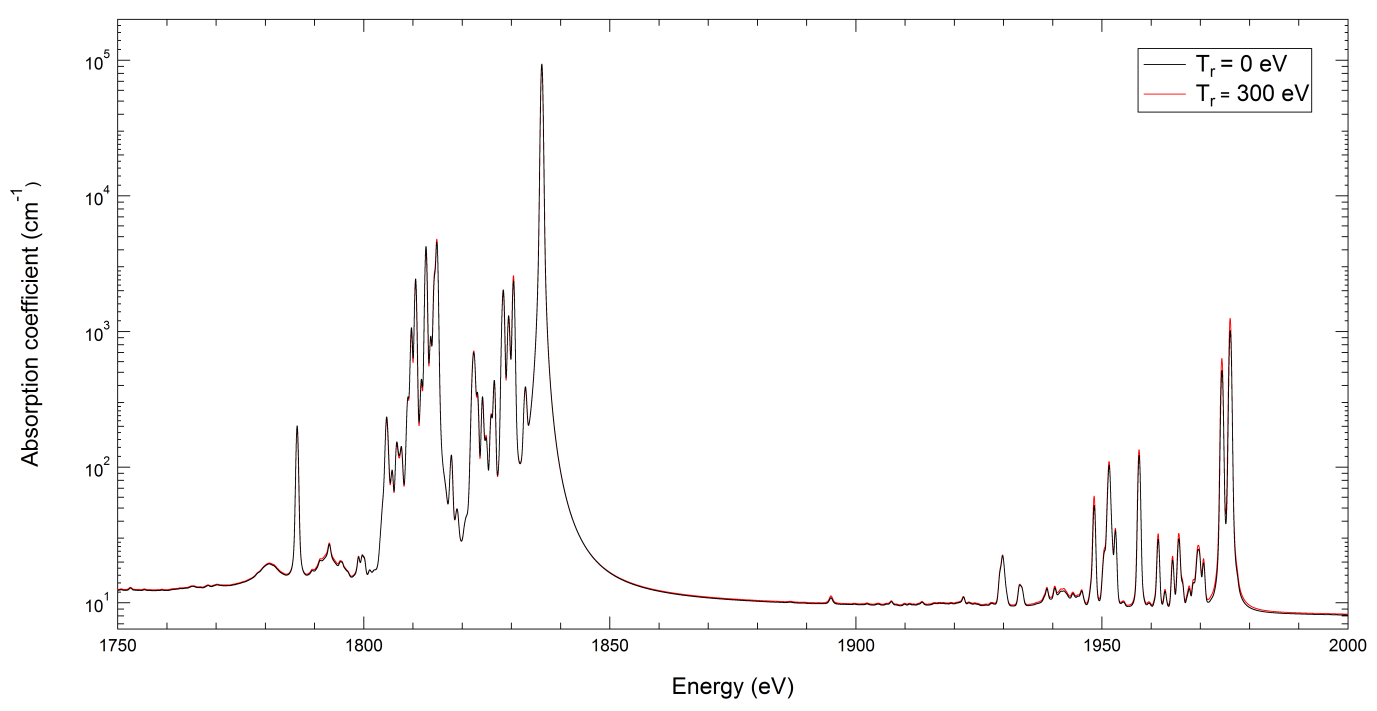

Fig. 6. Effect of the radiation field on the absorption of the $\mathrm{C}-\mathrm{Si}$ mixture. $T_{e}=300$ $\mathrm{eV}$ and $N_{e}=2 \times 10^{23} \mathrm{~cm}^{-3}$.

\section{ACKNOWLEDGMENTS}

We acknowledge the access to the cluster facility GMPCS of LUMAT (FR LUMAT 2764).

\section{References}

[1] M. F. Gu, Can. J. Phys. 86, 675 (2008).

[2] D. H. Sampson and H. L. Zhang, Astrophys. J. 335, 516 (1988).

[3] D. H. Sampson and H. L. Zhang, Phys. Rev. A 45, 1556 (1992).

[4] G. Xiong, J. Yang, J. Zhang, Z. Hu, Y. Zhao, B. Qing, G. Yang, M. Wei, R. Yi, T. Song, H. Li, Z. Yuan, M. Lv, X. Meng, Y. Xu, Z. Wu, and J. Yan, Astrophys. J. 816, 36 (2016).

[5] Q. Porcherot, J.-C. Pain, F. Gilleron, and T. Blenski, High Energy Density Phys. 7, 234 (2011).

[6] J.-C. Pain and F. Gilleron, High Energy Density Phys. 15, 30 (2015).

[7] J.-C. Pain, F. Gilleron, and T. Blenski, Laser Part. Beams 33, 201 (2015).

[8] T. Blenski, A. Grimaldi, and F. Perrot, J. Quant. Spectrosc. Radiat. Transfer 65, 91 (2000).

[9] R. D. Cowan, The theory of atomic structure and spectra (University of California Press, Berkeley, California, 1981).

[10] F. Perrot and T. Blenski, J. Phys. IV (France) 10, 474 (2000).

[11] J. Bauche, C. Bauche-Arnoult, and M. Klapisch, Phys. Rev. A 20, 2024 (1979).

[12] J. Bauche, C. Bauche-Arnoult, and M. Klapisch, Phys. Rev. A 25, 2641 (1982). 
[13] J. Bauche, C. Bauche-Arnoult, and M. Klapisch, Phys. Rev. A 31, 2248 (1985).

[14] C. A. Iglesias and V. Sonnad, High Energy Density Phys. 8, 133 (2012).

[15] A. Bar-Shalom, J. Oreg, W. H. Goldstein, D. Shvarts, and A. Zigler, Phys. Rev. A 40, 3183 (1989).

[16] W. Jarrah, D. Benredjem, J.-C. Pain, and J. Dubau, High Energy Density Phys. 24, 64 (2017).

[17] J. C. Stewart and K. D. Pyatt, Astrophys. J. 144, 1203 (1966).

[18] D. J. Hoarty, Phys. Rev. Lett. 110, 265003 (2013).

[19] G. Ecker and W. Kröll, Phys. Fluids 6, 62 (1963).

[20] M. Belkhiri, Plasma out of thermodynamical equilibrium: influence of the plasma environment on atomic structure and collisional cross sections, Ph.D. thesis, Université Paris-Sud, Orsay (2014).

[21] G. Massacrier and J. Dubau, J. Phys. B : At. Mol. Opt. Phys. 23, 2459 (1990).

[22] X. Li and F. B. Rosmej, EPL. 99, 33001 (2012).

[23] D. Benredjem, H. Nguyen, and G. Coulaud, J. Quant. Spectrosc. Radiat. Transfer 43, 415 (1990).

[24] H. Van Regemorter, Astrophys. J. 136, 906 (1962).

[25] http://aphysics2.lanl.gov/tempweb/lanl/2015 (2015).

[26] H. A. Kramers, Phil. Mag. Ser. 46, 836 (1923).

[27] R. M. More, J. Quant. Spectrosc. Radiat. Transfer 27, 345 (1982).

[28] X. Li, J. Yang, J. Zhang, Y. Zhao, G. Xiong, Z. Hu, and G. Yang, J. Phys. B: At. Mol. Opt. Phys. 50, 045701 (2017).

[29] E. Hill and S. Rose, High Energy Density Phys. 8, 307 (2012).

[30] S. Haan et al., UCRL-Conf-200359 (2003). 